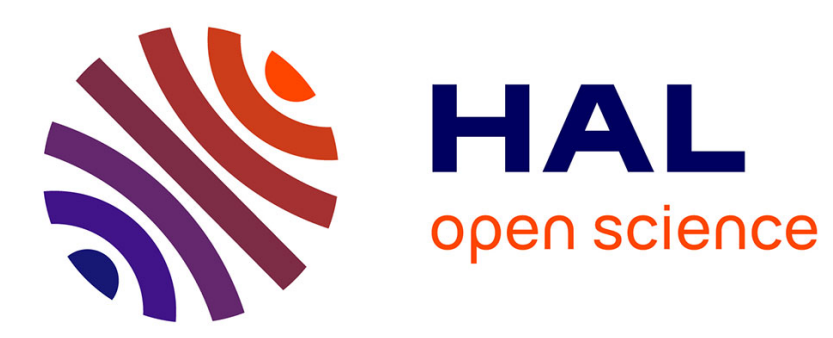

\title{
Persistent spins in the linear diffusion approximation of phase ordering and zeros of stationary gaussian processes
}

\author{
Bernard Derrida, Vincent Hakim, Reuven Zeitak
}

\section{To cite this version:}

Bernard Derrida, Vincent Hakim, Reuven Zeitak. Persistent spins in the linear diffusion approximation of phase ordering and zeros of stationary gaussian processes. Physical Review Letters, 1996, 77 (14), pp.2871-2874. 10.1103/PhysRevLett.77.2871 . hal-03285607

\section{HAL Id: hal-03285607 https://hal.science/hal-03285607}

Submitted on 21 Jul 2021

HAL is a multi-disciplinary open access archive for the deposit and dissemination of scientific research documents, whether they are published or not. The documents may come from teaching and research institutions in France or abroad, or from public or private research centers.
L'archive ouverte pluridisciplinaire HAL, est destinée au dépôt et à la diffusion de documents scientifiques de niveau recherche, publiés ou non, émanant des établissements d'enseignement et de recherche français ou étrangers, des laboratoires publics ou privés. 


\title{
Persistent Spins in the Linear Diffusion Approximation of Phase Ordering and Zeros of Stationary Gaussian Processes
}

\author{
Bernard Derrida, ${ }^{1,2}$ Vincent Hakim, ${ }^{1}$ and Reuven Zeitak ${ }^{1}$ \\ ${ }^{1}$ Laboratoire de Physique Statistique, Ecole Normale Supérieure, 24 rue Lhomond, 75231 Paris Cedex 05, France \\ ${ }^{2}$ Service de Physique Théorique, CE Saclay, F91191 Gif sur Yvette, France
}

(Received 29 May 1996)

\begin{abstract}
The fraction $r(t)$ of spins which have never flipped up to time $t$ is studied within a linear diffusion approximation to phase ordering. Numerical simulations show that $r(t)$ decays with time like a power law with a nontrivial exponent $\theta$ which depends on the space dimension. The dynamics is a special case of a stationary Gaussian process of known correlation function. The exponent $\theta$ is given by the asymptotic decay of the probability distribution of intervals between consecutive zero crossings. An approximation based on the assumption that successive zero crossings are independent random variables gives values of $\theta$ in close agreement with the results of simulations. [S0031-9007(96)01325-7]
\end{abstract}

PACS numbers: 02.50.-r, 05.40.+j, 82.20.-w

Phase ordering and domain growth in systems quenched from a disordered phase to a two-phase coexistence region has been for a long time a subject of active research [1]. Recently, a new facet of this problem has been uncovered [2-7]. For an Ising model initially in a random configuration, and evolving according to zero temperature Glauber dynamics, the fraction $r(t)$ of spins which have never flipped up to time $t$ decreases like a power law [2,3]

$$
r(t) \sim t^{-\theta} .
$$

Numerical data indicate that the exponent $\theta$ is nontrivial and varies with the dimension of space [2,3]. Similar exponents have been found in reaction-diffusion models with particles of two different mobilities [5,7,8]. The decay (1) has also been observed for the fraction of surface that has never been wet in an experiment on the growth of breath figures [9].

The simplicity of Glauber dynamics and its relation to soluble voter models has made possible an exact determination of $\theta$ for 1D Ising and Potts models [10]. In higher dimensions only numerical simulations $[3,11]$ or approximate methods [12] have been used so far. A very simple (and rather successful) description of coarsening for a nonconserved order parameter was proposed some time ago by Ohta, Jasnow, and Kawasaki (OJK) [13]. In this OJK approximation, the order parameter configuration $\phi(\vec{r}, t)$ a time $t$ after the quench, is the sign of a Gaussian field, initially random with zero average, which evolves according to a linear diffusion equation. Namely,

$$
\phi(\vec{r}, t)=\operatorname{sgn}[A(\vec{r}, t)]
$$

with

$\partial_{t} A(\vec{r}, t)=\nabla^{2} A(\vec{r}, t)$ and $\left\langle A(\vec{r}, 0) A\left(\vec{r}^{\prime}, 0\right)\right\rangle=\delta^{d}\left(\vec{r}-\vec{r}^{\prime}\right)$.

In this Letter, we first report numerical simulations showing that even within this approximation, the frac- tion $r(t)$ of persistent spins decays (1) with a nontrivial $\theta$ which varies with the spatial dimension $d$. We then show that determining $r(t)$ for the OJK dynamics is a particular case of a first-passage problem for correlated random variables. In a logarithmic time scale, these problems can be mapped onto stationary Gaussian processes. The exponent $\theta$ is given by the asymptotic decay of the distribution of intervals between zero crossings for the associated process. To our surprise, given the correlation function of the Gaussian process, the determination of this asymptotic decay turns out to be a hard unsolved problem [14-16]. We propose here a simple approximation which consists in taking the lengths of successive intervals between zero crossing as independent identically distributed random variables. The probability distribution of the interval lengths is then chosen so as to reproduce the known sign-correlation function of the stationary Gaussian process [17].

We have simulated the diffusion equation (3) by using a finite difference first-order forward Euler scheme on cubic lattices in $d=1,2,3$

$$
A_{i}(t+\Delta t)=(1-2 d \Delta t) A_{i}(t)+\Delta t \sum_{\langle i j\rangle} A_{j}(t) .
$$

We used mostly $\Delta t=0.1$ on lattices of up to $10^{6}$ sites and, in a single run, we measured $r(t)$, the fraction of lattice sites $i$ for which $A_{i}$ never changed sign up to time $t$ (as long as $\sqrt{t}$ is much less than the linear size of the system, finite size effects are expected to be negligible). Our results are shown in Fig. 1 and indicate that $r(t)$ decays as in (1) with $\theta \simeq 0.12$ in $d=1, \theta \simeq 0.18$ in $d=2$, and $\theta \simeq 0.23$ in $d=3$. Other choices of $\Delta t$ indicate that $\theta$ is not affected by the value of $\Delta t$ (as long as $\Delta t<1 / 2 d$ to ensure the stability of the Euler scheme).

The stochastic process at a particular lattice point (the origin, say) is of the type

$$
A(t)=\sum_{x>0} K(x, t) \eta_{x},
$$




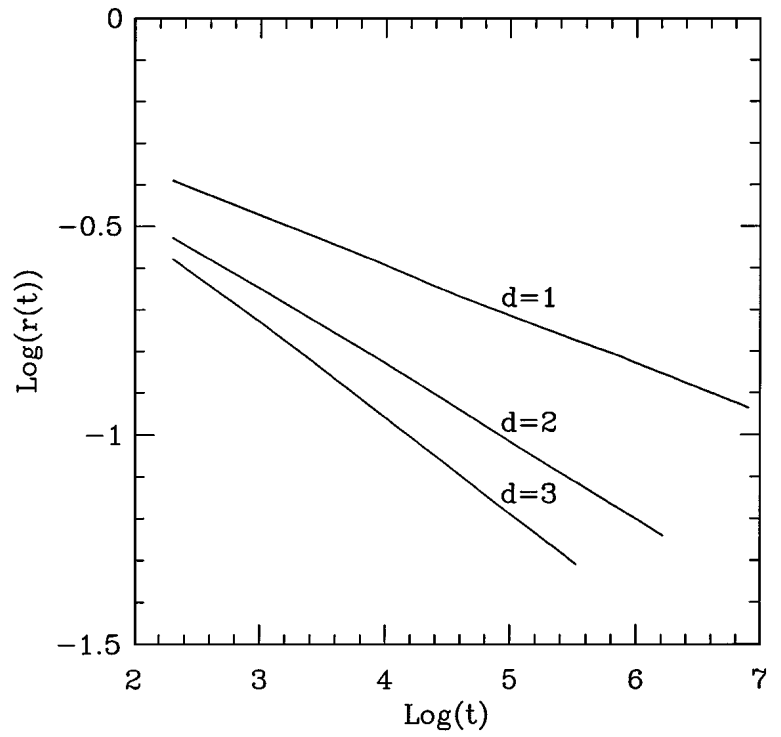

FIG. 1. Log-log plot of the fraction $r(t)$ of sites $i$ for which $A_{i}$ has never changed sign vs time for the discretized diffusion equation in dimension $d=1,2,3 . \quad r(t)$ shows a power-law decay with an exponent which depends on $d$.

where the $\eta_{x}$ are random Gaussian variables (with $\left\langle\eta_{x}^{2}\right\rangle=$ $1)$ and the kernel $K(x, t)$ is

$$
K(x, t)=\text { const } \times x^{(d-1) / 2} \exp \left(-x^{2} / 4 t\right) /(4 \pi t)^{d / 2} .
$$

Other examples with different kernels include usual random walks $\left(\dot{A}=\eta_{t}\right)$ for which $K$ is the Heavyside function $K(x, t)=H(t-x)$ and $\theta=1 / 2$, and walks under the influence of a random force $\left(\ddot{A}=\eta_{t}\right)$ for which $K(x, t)=(t-x) H(t-x)$ and $\theta=1 / 4[18,19]$. Clearly, the first passage exponent $\theta$ depends on the shape of the kernel $K(x, t)$ and takes different values for square, triangular, or diffusive kernels.

It is useful to note that (5) is a Gaussian process since it is a linear combination of random Gaussian variables. This process is therefore entirely characterized by its pair correlation function $\left\langle A(t) A\left(t^{\prime}\right)\right\rangle$. This is also true of the rescaled variables $X(t)=A(t) / \sqrt{\langle A(t) A(t)\rangle}$, which can be considered as well since we are only interested in the sign changes of $A(t)$. In the long time limit, the correlation function $\left\langle X(t) X\left(t^{\prime}\right)\right\rangle$ depends only on the time ratio $t / t^{\prime}$ for the process considered (6). In a logarithmic time scale, $u=\ln (t)$, if one defines $Y(u)=X(t), Y(u)$ is therefore a Gaussian stationary process

$$
\left\langle Y(u) Y\left(u^{\prime}\right)\right\rangle=C\left(u-u^{\prime}\right),
$$

with $C(u)=[1 / \cosh (u / 2)]^{d / 2}$ for the OJK process in dimension $d$ [and $C(u)=\exp (-|u| / 2)$ for the usual random walk]. $C$ is a rapidly decreasing function of $u$. Therefore $Y(u)$ and $Y(u+l)$ are effectively uncorrelated for sufficiently large $l$ and it is intuitively clear that the probability that $Y(u)$ keeps the same sign for an interval of length $l$ decreases exponentially with $l$ for large $l$
$\operatorname{Prob}\left\{Y\left(u+l^{\prime}\right)>0,0<l^{\prime}<l\right\} \sim \exp (-\theta l)$ for $l \gg 1$

and $\theta$ measures the (inverse) decorrelation time in the $u$ variable. The asymptotic behavior (8) implies that the probability of not flipping in the original $t$ variable from time $t_{1}$ to $t_{2}$ with $t_{1} \ll t_{2}$ is a power law $\left(t_{1} / t_{2}\right)^{\theta}$ as numerically observed above.

In order to compute $\theta$, one needs the large $l$ behavior of the distribution $p(l)$ of the lengths $l$ of intervals between consecutive zeros of a Gaussian stationary process characterized by a given $C(l)$. As observed by Rice more than forty years ago [14], this is a surprisingly hard problem which is still unsolved $[15,16,18]$. So one has to resort to approximate methods. Perturbative and variational techniques using the free Brownian walk as a starting point have been proposed recently $[12,20]$. However, the free Brownian walk and other similar Markovian processes have an infinite density of zero crossings and are not a good starting point for cases, for which $C^{\prime \prime}(0)$ is finite like the OJK process, which have a finite density of zero crossings [the density of zeros is given [14] by $\sqrt{-C^{\prime \prime}(0)} / \pi$ ] We propose here an approximation more suited to these cases which is inspired by recent works on the distribution of domain sizes in a 1D coarsening Ising model [21], where a similar approximation has been shown to be rather accurate [22]. It consists in considering the lengths between consecutive zero crossings as independent random variables, identically distributed according to a probability distribution $p(l)$. This $p(l)$ is determined by requiring that the sign correlation function is the same for the approximate independent interval approximation and for the original stationary Gaussian process [23].

For a process with correlation function $C(l)$, the probability $P_{+}(l)$ that $Y(u) Y(u+l)>0$ is given by [14]

$$
P_{+}(l)=\frac{1}{2}+\frac{1}{\pi} \arcsin [C(l)] .
$$

To obtain the corresponding probability for the independent interval process, it is convenient to determine first the probability $P_{\mathrm{cr}}(l)$ that a point at distance $l$ from an up crossing be positive. Either the first interval after the up crossing is longer than $l$, or the interval is of size $l_{1}<l$ and the point is positive with probability $1-P_{\mathrm{cr}}\left(l-l_{1}\right)$. So $P_{\mathrm{cr}}(l)$ satisfies

$$
\begin{aligned}
P_{\mathrm{cr}}(l)= & \int_{l}^{+\infty} d l_{1} p\left(l_{1}\right) \\
& +\int_{0}^{l} d l_{1} p\left(l_{1}\right)\left[1-P_{\mathrm{cr}}\left(l-l_{1}\right)\right] .
\end{aligned}
$$

Taking Laplace transforms (which we denote with tilde), one obtains

$$
\tilde{P}_{\text {cr }}(\lambda)=\{\lambda[1+\tilde{p}(\lambda)]\}^{-1} .
$$

This can now be used to determine the probability that $Y(u) Y(u+l)>0$ for the independent interval process. 
A given point is at a distance $l_{1}$ from the first crossing on its right, with probability

$$
Q\left(l_{1}\right)=\frac{\int_{l_{1}}^{+\infty} p(l) d l}{\int_{0}^{+\infty} l p(l) d l} \text { or } \tilde{Q}(\lambda)=\frac{\tilde{p}(\lambda)-1}{\lambda \tilde{p}^{\prime}(0)} .
$$

If $Y(u)$ and $Y(u+l)$ have the same sign either they belong to the same interval or $Y(u+l)$ has a sign opposite to points immediately to the right of the first crossing after $u$, so that

$$
\begin{aligned}
P_{+}(l)= & \int_{l}^{+\infty} d l_{1} Q\left(l_{1}\right) \\
& +\int_{0}^{l} d l_{1} Q\left(l_{1}\right)\left[1-P_{\mathrm{cr}}\left(l-l_{1}\right)\right] .
\end{aligned}
$$

This gives for the Laplace transforms

$$
\tilde{P}_{+}(\lambda)=1 / \lambda-\tilde{Q}(\lambda) \tilde{P}_{\mathrm{cr}}(\lambda) .
$$

Substituting (11) and (12) into (14), one finally relates the distribution $p(l)$ of interval lengths to the probability $P_{+}(l)$ that two points at distance $l$ have the same sign for the independent interval process (or rather their Laplace transforms)

$$
\tilde{p}(\lambda)=\frac{1-\left[\lambda^{2} \tilde{P}_{+}(\lambda)-\lambda\right] \tilde{p}^{\prime}(0)}{1+\left[\lambda^{2} \tilde{P}_{+}(\lambda)-\lambda\right] \tilde{p}^{\prime}(0)} .
$$

For an integrable distribution of interval lengths, $\tilde{p}(\lambda)$ should tend to zero as $\lambda \rightarrow+\infty$. Using (15), this gives $\tilde{p}^{\prime}(0)=1 / P_{+}^{\prime}(0)$, which simply means that the mean interval size $\left[-\tilde{p}^{\prime}(0)\right]$ is the inverse of the density of zeros $\left[-P_{+}^{\prime}(0)\right]$.

Equation (15) would be exact if the successive intervals were uncorrelated. Here the heart of the approximation is to choose the exact $P_{+}(l)$ given by (9) for a given process and determine $p(l)$ through (15). The large $l$ behavior of $p(l)$ is given by the singularity of its Laplace transform with the largest (negative) real part, i.e., by the largest zero of $P_{+}^{\prime}(0)=\lambda-\lambda^{2} \tilde{P}_{+}(\lambda)$ or, using (9),

$$
\sqrt{-C^{\prime \prime}(0)}=-\theta \int_{0}^{+\infty} d l \exp (\theta l) C^{\prime}(l)\left[1-C^{2}(l)\right]^{-1 / 2} .
$$

In the OJK case, $C(l)=[1 / \cosh (l / 2)]^{d / 2}$. A numerical solution of (16) gives $\theta \simeq 0.1203$ for $d=1, \theta \simeq 0.186$ for $d=2$, and $\theta \simeq 0.2358$ for $d=3$ in very good agreement with the previous numerical results. For large $d$ the independent interval approximation (16) gives $\theta \sim \kappa \sqrt{d}$ with $\kappa \simeq 0.1455$ [24]. Note that, as $C(l)=$ $\exp (-|l| / 2)$ for the case of a free Brownian motion, $C^{\prime \prime}(0)=\infty$ and the only possibility to satisfy (16) is that the integral on the right-hand side diverges, leading to $\theta=1 / 2$ which is exact.

The whole distribution $p(l)$ can also be obtained by inverting the Laplace transform. One can obtain the Taylor expansion of $p(l)$ around $l=0$ easily from (15) and then draw $p(l)$ using Padé approximants [25]. The obtained shapes for the diffusive case in $d=1,2,3$ are plotted in Fig. 2 and compared with results of direct simulations of the stationary Gaussian processes.

We have shown that nontrivial exponents for the number of persistent spins appear even in the simple OJK approximation of coarsening. Contrarily to the case of Glauber dynamics [3,4], $\theta$ increases with dimension. This means the OJK theory is too crude an approximation to predict $r(t)$ for Glauber dynamics. It is remarkable that the independent interval approximation $[21,22]$ gives here such accurate predictions for the OJK exponents. Still, it would be interesting to better understand it, for example, by a maximum entropy argument. Of course, it would be also interesting to estimate the correlations $p\left(l_{1}, l_{2}\right)$ between successive intervals and see whether one could develop a systematic method of including these correlations. Another issue would be to see under what conditions the distributions $p(l)$ obtained via (15) are positive for general kernels $K$ (as nothing guarantees a priori that it is so).

We have considered here a first passage problem at the threshold zero in the case where the initial Gaussian field $A(\vec{r}, t)$ has a zero average. If this average initial field, or the threshold, was nonzero, $r(t)$ would not vanish in the long time limit. This is in contrast with the 1D Ising model where $r(t)$ vanishes even for a nonzero initial magnetization with a first-passage exponent which depends on this initial magnetization $[4,10]$. There are also interesting examples where the threshold depends on time [26]. It would be interesting to extend the independent interval approximation to such cases. Lastly, one could try to extend the OJK approximation to cases where the order parameter is more complicated than a

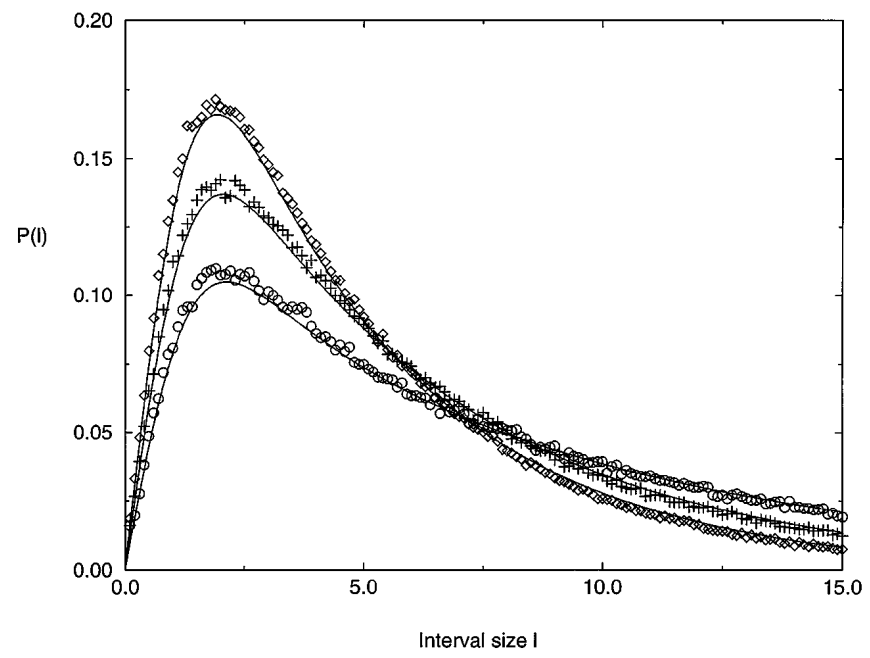

FIG. 2. The probability distribution function of having an interval of length $l$ for the Gaussian stationary process associated to the diffusion equation in $d=1,2,3$ as obtained in the independent interval approximation (lines) and by direct simulations of the Gaussian processes $(d=1$ circles, $d=2$ pluses, $d=3$ diamonds) in Fourier space (1000 realizations of length 3276.8 ; bin size 0.1 ). 
scalar (as in the Potts model) to see how the above approximation can be adapted to such cases.

We are grateful to $\mathrm{D}$. Dhar for many enlightening discussions on the subject of this paper when two of us (B.D. and V.H.) were visiting the Newton Institute (Cambridge, UK). We also thank J.P. Bouchaud for interesting comments.

[1] J. Langer, in Solids Far from Equilibrium, edited by C. Godrèche (Cambridge Univ. Press, Cambridge, 1992); A. J. Bray, Adv. Phys. 43, 357 (1994).

[2] B. Derrida, A. J. Bray, and C. Godrèche, J. Phys. A 27, L357 (1994).

[3] D. Stauffer, J. Phys. A 27, 5029 (1994).

[4] A. J. Bray, B. Derrida, and C. Godrèche, Europhys. Lett. 27, 175 (1994).

[5] P.L. Krapivsky, E. Ben-Naim, and S. Redner, Phys. Rev. E 50, 2474 (1994).

[6] P. L. Krapivsky, S. Redner, and F. Leyvraz, Phys. Rev. E 51, 3977 (1995).

[7] E. Ben-Naim, L. Frachebourg, and P. L. Krapivsky, Phys. Rev. E 53, 3078 (1996); (to be published).

[8] J. L. Cardy, J. Phys. A 28, L19 (1995).

[9] M. Marcosmartin, D. Beysens, J.P. Bouchaud, C. Godrèche, and I. Yekutieli, Physica (Amsterdam) 214A, 396 (1995).

[10] B. Derrida, V. Hakim, and V. Pasquier, Phys. Rev. Lett. 75, 751 (1995); J. Stat. Phys. (to be published).

[11] B. Derrida, P. M. C. de Oliveira, and D. Stauffer, Physica (Amsterdam) 224A, 604 (1996).

[12] S. N. Majumdar and C. Sire, Phys. Rev. Lett. 77, 1420 (1996).

[13] T. Ohta, D. Jasnow, and K. Kawasaki, Phys. Rev. Lett. 49, 1223 (1982).

[14] S. O. Rice, Bell Syst. Tech. Journal 23 and 24 [reprinted in N. Wax, Selected Papers on Noise and Stochastic Processes (Dover, New York, 1954)].
[15] M. Kac, SIAM Rev. 4, 1 (1962).

[16] H. Cramer and M. R. Leadbetter, Stationary and Related Stochastic Processes (Wiley, New York, 1967).

[17] After an oral presentation of our results at the '96 Nordic Stat. Phys. Workhop in Copenhagen, A. Bray informed us that results similar to ours have been obtained independently in S. N. Majumdar, C. Sire, A. J. Bray, and S. J. Cornell, preceding Letter, Phys. Rev. Lett. 77, 2867 (1996).

[18] H. P. McKean Jr., J. Math. Kyoto Univ. 2, 227 (1963); M. Goldman, Ann. Math. Stat. 42, 2151 (1971).

[19] Y. Sinai, Theor. Math. Phys. 90, 323 (1992); T. W. Burkhardt, J. Phys. A 26, L1157 (1993).

[20] V. Hakim and R. Zeitak (to be published).

[21] P. A. Alemany and D. ben-Avraham, Phys. Lett. A 206, 18 (1995).

[22] B. Derrida and R. Zeitak, Phys. Rev. E (to be published).

[23] It is mentioned in [17] that this approximation had been previously proposed for stationary Gaussian processes by J. A. McFadden, IRE Transaction on Information Theory 4, 14 (1957).

[24] The constant $\kappa$ is solution of the equation $2 \sqrt{2}=$ $\kappa \int_{0}^{+\infty} d u \exp (\kappa u) u / \sqrt{\exp \left(u^{2} / 8\right)-1}$.

[25] $\tilde{p}(\lambda)$ was expanded in powers of $1 / \lambda^{2}$ using (15). This provided the Taylor series of $p(l)$ around $l=0$ which consists only of odd powers of $l$ with coefficients of alternating signs [for example, $p(l)=l / 2-3 l^{3} / 8+$ $15 l^{5} / 64+\cdots$, for $\left.d=2\right]$. This series was resummed using Padé approximants of the form $l A\left(l^{2}\right) / B\left(l^{2}\right)$ with $A$ and $B$ polynomials of degree $N$ and $N+1(N=11$ is used for the curves of Fig. 2). $N$ was chosen large enough so, in the range of $l$ of interest, changing $N$ did not noticeably change the curve shown. It was checked that the decaying part of the curves were straight in a log-linear plot with slopes in agreement with the direct numerical solution of (16).

[26] P.L. Krapivsky and S. Redner, Am. J. Phys. 64, 546 (1996). 